\title{
El hábitat popular hoy en las ciudades peruanas. Una contribución a los estudios urbanos en el Perú
}

Manuel Dammert Guardia, Jérémy Robert y Pablo Vega Centeno

\section{OpenEdition}

Edición electrónica

URL: http://journals.openedition.org/bifea/8543

DOI: $10.4000 /$ bifea. 8543

ISSN: 2076-5827

Editor

Institut Français d'Études Andines

Edición impresa

Fecha de publicación: 8 diciembre 2017

Paginación: 405-412

ISSN: 0303-7495

\section{Referencia electrónica}

Manuel Dammert Guardia, Jérémy Robert y Pablo Vega Centeno, «El hábitat popular hoy en las ciudades peruanas. Una contribución a los estudios urbanos en el Perú ", Bulletin de l'Institut français d'études andines [En línea], 46 (3) | 2017, Publicado el 08 diciembre 2017, consultado el 06 noviembre 2020. URL : http://journals.openedition.org/bifea/8543 ; DOI : https://doi.org/10.4000/bifea.8543

us du Bulletin de l'Institut français d'études andines sont mis à disposition selon les termes de la licence Creative Commons Attribution - Pas d'Utilisation Commerciale - Pas de Modification 4.0 International. 


\title{
El hábitat popular hoy en las ciudades peruanas. Una contribución a los estudios urbanos en el Perú
}

\author{
Manuel Dammert Guardia* \\ Jérémy Robert** \\ Pablo Vega Centeno ${ }^{* * *}$
}

\section{ELEMENTOS DE CONTEXTO}

Este volumen busca contribuir a la dinámica de los estudios urbanos peruanos. Reúne investigaciones sobre el hábitat popular, objeto tradicional de los estudios urbanos, el cual sigue siendo uno de los mayores desafíos de las ciudades del país: de acuerdo con el Ministerio de Vivienda, Construcción y Saneamiento (MVCS, 2012), el 73\% de los núcleos urbanos en Lima Metropolitana puede ser considerado como Barrio Urbano Marginal (BUM)1. Esta cifra es mayor que en otras ciudades del país donde los BUM alcanzan, sin embargo, más de la mitad del espacio urbanizado (de 50 a 60\%).

* Doctorando del Centro de Estudios Sociológicos (CES) de El Colegio de México. E-mail: manueldammert@gmail.com

** Investigador del Instituto Francés de Estudios Andinos - IFEA (UMIFRE 17 MEAE / CNRS USR 3337 América Latina). E-mail: jeremy.robert@cnrs.fr

${ }^{* * *}$ Investigador del Centro de Investigación de la Arquitectura y la Ciudad (CIAC) de la Pontificia Universidad Católica del Perú (PUCP). E-mail: pvega@pucp.edu.pe

1 Para el Ministerio de Vivienda, Construcción y Saneamiento, el BUM está «caracterizado por presentar altos niveles de pobreza monetaria y no monetaria, y carecer, total o parcialmente, de servicios de infraestructura y servicios de equipamiento» (MVCS, 2012: 6). 
Los artículos que aquí se presentan son el resultado de las discusiones que se iniciaron en septiembre del año 2016, en el marco del Foro Urbano Nacional «El hábitat popular en debate: las ciudades peruanas en perspectiva ${ }^{2}$. Este evento, coorganizado por el IFEA y el CIAC-PUCP, se inscribe dentro de una dinámica de colaboración entre estas dos instituciones con el principal objetivo de promover y contribuir al desarrollo de los estudios urbanos en el Perú. Asimismo, se inserta en una dinámica regional de intercambios — en su mayoría seminarios internacionales_ buscando estructurar una red entre académicos, en particular a nivel regional, en Colombia, Ecuador, Perú y Bolivia. La publicación del libro La cuestión urbana en la región andina. Miradas sobre la investigación y la formación (Metzger et al., eds., 2016) es uno de los resultados de esta cooperación. Este trabajo permitió hacer un balance de los grandes temas de investigación en la región e identificar posibilidades de discusión entre países acerca de dinámicas comunes. Al mismo tiempo permitió evidenciar carencias. En el Perú, por ejemplo, la gran cantidad de estudios sobre la ciudad de Lima contrasta con la escasez de trabajos conocidos sobre otros fenómenos urbanos del país (Calderón \& Vega Centeno, 2016). Es así que surgió la idea de explorar qué se hacía en términos de investigación urbana en el país, en Lima y sobre todo fuera de la capital.

Bajo esta perspectiva se organizó el Foro Urbano Nacional, con el objetivo de debatir sobre el hábitat popular hoy en las ciudades peruanas. Este foro buscó reunir estudios y trabajos de investigación recientes realizados en distintas ciudades del Perú, con el fin de generar un espacio de discusión nacional. La elección del tema del «hábitat popular» respondió a dos argumentos. Primero porque era suficientemente amplio para convocar una gran cantidad de trabajos, en la perspectiva de interesar a los investigadores fuera de la ciudad de Lima, y para construir análisis comparativos. El segundo argumento era aprovechar la coyuntura de la reunión de Hábitat III en Quito, en octubre de 2016, donde el «hábitat popular» fue el tema central. En este marco, la convocatoria planteó interrogantes relativamente abiertas: ¿Cómo se discute y aborda hoy la cuestión del hábitat en las ciudades peruanas? ¿Cuáles son los cambios en la manera de enfocar y trabajar la cuestión del hábitat popular?

Si bien el evento de 2016 logró una gran convocatoria, con más de 40 ponentes y un público numeroso, el objetivo de «representación nacional» no fue alcanzado sino de manera parcial. Participaron investigadores de Arequipa, Puno y Huancayo, pero desafortunadamente no lograron presentar resultados de investigación. Esta ausencia es compensada en este volumen, en parte, con la

2 Este seminario fue parte de un evento mayor, coordinado por el IFEA en el año 2016, titulado «¿ंCiudades populares, ciudades sostenibles? El hábitat popular en debate», y realizado en colaboración con varias instituciones, en particular el Centro de Investigación de la Arquitectura y la Ciudad (CIAC) de la Pontificia Universidad Católica del Perú (PUCP), la Embajada de Francia, el Institut de recherche pour le développement (IRD), la Alianza Francesa de Lima y de Arequipa, I'Insitut Français, el Centro de Estudios y Promoción del Desarrollo (DESCO), Hábitat International Coalition (HIC) y Cooperazione Internazionale (COOPI). Para mayor información consúltese el blog https://ifea.hypotheses.org/752. 
selección de dos textos de investigadores limeños sobre ciudades menores. El escaso desarrollo de estudios fuera de Lima pone en evidencia la necesidad de reforzar las investigaciones en temas urbanos en provincia con las universidades locales y de impulsar dinámicas de intercambio y colaboración. Es más necesario aún en vista de la demanda existente, tanto por parte de los estudiantes (lo que se evidenció en el debate descentralizado llevado a cabo en Arequipa en el marco del Foro Urbano Nacional, en septiembre de 2016) como para la acción pública en ciudades con evoluciones muy fuertes y rápidas.

A pesar de las dificultades se puede abordar el futuro con buenos augurios. En el evento de 2016 se pudo observar la vitalidad de la investigación urbana, admirablemente representada en este volumen por cinco textos de jóvenes investigadores y uno en coautoría de una investigadora experimentada y de una joven investigadora. Esta característica mayor deja entrever estimulantes perspectivas para los estudios urbanos en el país.

Con el fin de introducir este dossier, mencionaremos brevemente a las grandes líneas del debate acerca del hábitat popular en el Perú antes de presentar los textos seleccionados.

\section{DEBATES SOBRE EL HÁBITAT POPULAR EN EL PERÚ}

El hábitat popular, en particular a través de los mecanismos populares de acceso al suelo y a la vivienda, ha sido uno de los ejes de discusión privilegiados en la investigación urbana en el Perú (Calderón \& Vega Centeno, 2016). Los trabajos desarrollados siguieron diferentes perspectivas analíticas, desde la óptica de la vivienda popular y su carácter progresivo (Turner \& Fichter, coords., 1976), la configuración socioespacial en torno a los asentamientos humanos o barriadas (Degregori et al., 1986; Driant, 1991), hasta la forma en que esta ciudad «ilegal» (Calderón Cockburn, 2005) o «popular» (Riofrío, 1991) expresa los límites o debilidades del Estado para garantizar servicios (Matos Mar, 2004). Siguiendo mayormente una visión dualista, oponiendo lo formal y lo informal (aunque se reconozca la permeabilidad de las fronteras), estos estudios se dedicaron a caracterizar los procesos de acceso informal al suelo, de construcción progresiva de vivienda y de consolidación urbana. Estos mecanismos han estado asociados a cuatro grandes narrativas, bastante vinculadas entre sí:

- En primer lugar, se encuentran los textos fundacionales de J. Turner sobre la vivienda progresiva y sobre cómo los sectores populares movilizan una serie de recursos. Frente a una visión que atribuía rasgos morales y tipos específicos de conducta a los sectores populares (asociados a la delincuencia), Turner abogaba por una visión positiva de las barriadas en Lima. Su propuesta era reconocer el proceso innovador de las barriadas y la capacidad movilizadora e integradora de las organizaciones barriales, y apostar por que los sectores populares desarrollaran progresivamente su vivienda. Esta propuesta tuvo importantes consecuencias en la manera de comprender las barriadas y 
las políticas públicas. En este contexto, «la construcción de proyectos de vivienda pública se reemplazó por proyectos de lotes con servicios, es decir, lotes distribuidos con un mínimo de infraestructuras básicas necesarias, pero aptos para el desarrollo progresivo por los mismos residentes» (FernándezMaldonado, 2015: 7).

- Una segunda narrativa propone asumir la existencia de un criterio de diferenciación en torno a un esquema dualista de «formal e informal» (Calderón Cockburn, 2005). Para autores como de Soto (1987), existe una asociación entre la informalidad laboral y la informalidad urbana. Estas informalidades serían la principal razón de acumulación de recursos, pero al mismo tiempo la principal traba para el mejoramiento de las condiciones de vida. Por tal motivo, uno de los ejes centrales de la política pública urbana en el país ha sido la «formalización» (a través de programas como COFOPRI). En una orilla distinta, Calderón Cockburn (2005) sugiere que esta informalidad urbana no es el resultado de la ausencia del Estado, sino que muchas veces es promovida, auspiciada y confirmada por el Estado a través de sus políticas acerca de las barriadas, el suelo y la vivienda.

- La tercera narrativa fundacional plantea comprender las barriadas y el hábitat popular como expresión de un nuevo orden institucional. Frente a la incapacidad del Estado para garantizar mecanismos de integración social (a través de servicios, titulación y acceso a la vivienda), los sectores populares habrían generado una nueva institucionalidad (Matos Mar, 2004). En términos generales, se considera que la informalidad forma parte de un complejo entramado institucional que opera en los márgenes del Estado, modificándolo, y reorganizando también las características de aquello que definimos como «formal» en términos urbanos (mercados regulados de acceso al suelo).

- La cuarta narrativa, principalmente de tipo cultural, asume que existe una relación entre la configuración socioespacial (barriada, ciudad popular, etc.) y los rasgos morales, o de comportamiento de los individuos y las colectividades. Este tipo de aproximación, muy vinculada al desarrollo de una perspectiva de la cultura de la pobreza, propone - a su vez- que la degradación o precariedad material está asociada a ciertos valores. Pese a ser muy criticada, esa visión sigue siendo importante en la opinión y en el debate públicos.

Estas aproximaciones analíticas al hábitat popular han conocido evoluciones con cambios simultáneos entre los procesos sociales y las matrices de comprensión. Así, se pueden identificar diferentes «momentos» en la configuración de las barriadas y del hábitat popular (Barreda \& Ramírez Corzo, 2004). Implica no asumir el hábitat popular como figuras socioespaciales homogéneas, sino considerar la superposición de «nuevos y viejos» procesos, y reconocer su heterogeneidad en términos socioeconómicos y de trayectorias de consolidación urbana.

Más allá de estos apuntes, las dinámicas actuales del hábitat popular invitan a una reactualización de las perspectivas analíticas. En los últimos años, las lógicas de expansión urbana se han transformado con el aumento del tráfico 
de terrenos y de prácticas delictivas y violentas. Otro cambio es la disminución del rol de las organizaciones de escala barrial y/o vecinal, y los bajos niveles de involucramiento y participación de los residentes en ellas o en la vida colectiva de sus entornos territoriales. Los progresos alcanzados en el acceso a la titulación de la propiedad y a los servicios básicos (luz, agua y saneamiento), que son objeto de demandas colectivas y logran agrupar intereses, son unos de los factores de este debilitamiento. A medida que avanzan los procesos de consolidación urbana, las acciones colectivas y organizadas parecen haber perdido importancia. Sin embargo, surgen nuevas demandas con dinámicas distintas hacia la movilidad, el espacio público y la seguridad ciudadana. Más allá de las visiones positivas (que destacan la solidaridad, la comunidad o el emprendimiento de los sectores urbanopopulares) o negativas (que atribuyen a los residentes la propia responsabilidad de sus problemas), parece más pertinente interrogar cómo el actual modelo de producción del hábitat popular (en tanto configuración socioespacial) y del espacio urbano en su conjunto está vinculado a cambios en los patrones de interacción, integración y organización de la acción colectiva. Estas dinámicas, entre otras, invitan a cuestionar el rol y la presencia del Estado en estos espacios. En esta línea, los investigadores han orientado su interés hacia las lógicas de la inversión pública (Espinoza \& Fort, 2017) o de los programas de mejoramiento y de integración de los barrios, como ha sido el caso de Barrio Mío de la Municipalidad Metropolitana de Lima (Molnárová et al., eds., 2017).

En este contexto de nuevas dinámicas de producción y gobernanza de estos espacios urbanos, la dicotomía entre lo formal y lo informal deja de ser el rasgo principal de diferenciación, por un lado, frente a procesos de consolidación duraderos y, por otro lado, frente a prácticas delictivas. Estas dinámicas cuestionan no solamente a las autoridades públicas, sino también invitan al investigador a descifrarlas. Demuestran sin ninguna duda que el hábitat popular en el Perú no ha dejado de ser un tema central de preocupación. Los textos que se presentan en este volumen contribuyen a alimentar esta agenda de investigación, aportando miradas originales tanto sobre la organización y las dinámicas de transformación del hábitat popular como sobre las organizaciones y la cohesión social, o finalmente, sobre los procesos de urbanización y la planificación territorial.

\section{LOS TEXTOS}

La entrevista a Jean-Claude Driant, quien participó en el Foro Urbano en septiembre de 2016, introduce el debate y al mismo tiempo abre nuevas líneas de discusión. Su regreso a Lima, treinta años después de su estadía en el IFEA para el desarrollo de su investigación doctoral sobre las barriadas de la ciudad, ha sido una oportunidad para recordar la larga tradición de investigaciones francesas sobre el tema urbano en el Perú. De la misma manera, ha sido una ocasión para dialogar sobre distintas experiencias francesas y peruanas: una apuesta a romper fronteras y a la apertura de nuevas discusiones. A partir de las dinámicas del hábitat popular en Francia, Jean-Claude Driant evidencia tanto similitudes como diferencias. Los 
debates acerca de la «mixidad» en Francia o de la intervención del Estado sobre el mercado inmobiliario tienen su paralelo (y diferencias) en el Perú. Sin embargo, el tema de la sostenibilidad, que orienta con fuerza la política pública francesa, encuentra muy poca resonancia en la realidad peruana.

Los cuatro textos siguientes se inscriben en la línea de los trabajos clásicos sobre el hábitat popular, aportando cada uno nuevas perspectivas de análisis. Daniel Ramírez Corzo Nicolini propone discutir la pertinencia de la categoría de «asentamiento humano» (o, se podría decir, de «ciudad popular») al plantear que no podemos referirnos a estas configuraciones socioespaciales como las encargadas de su «autourbanización». Por el contrario, el autor propone abordar las relaciones entre los distintos actores del «espacio barrial» (vecinos, dirigentes, autoridades) como relaciones asimétricas. A partir del análisis de dichas relaciones, busca evidenciar las particularidades de cómo se «produce» el hábitat popular hoy en día, más allá de visiones estigmatizadoras. Destaca el rol central de la desconfianza y de la configuración de distancias sociales en la vida pública de los «asentamientos humanos». La relación entre «vecinos» y «dirigentes vecinales» aparece como un tema álgido que ilustra las dificultades de la acción colectiva, la organización y la participación. El capital social de los actores, los conflictos y las relaciones de poder cobran entonces mayor importancia para entender las dinámicas en curso.

Jimena Ñquen Castro-Pozo, en su artículo sobre trayectorias diferenciadas en Huaycán, visibiliza un tema central en el debate sobre el hábitat popular: la existencia de procesos de diferenciación que se evidencian dentro de las familias y entre generaciones de familiares en lo que concierne a los mecanismos de acceso al suelo y a la producción de la vivienda. Pone en el centro del debate a los procesos de consolidación urbana, discutiendo el rol tanto de las estrategias individuales y familiares como de los entramados institucionales y organizativos. Muestra también las relaciones diferenciadas de los habitantes con el espacio. Aquellas personas que estuvieron vinculadas a los momentos de «fundación» de un asentamiento o de una urbanización mantienen una relación distinta con este espacio en comparación con sus hijos. De la misma manera, evidencia estrategias diferentes si las personas se encuentran en lógicas de subsistencia y necesidad o, más bien, de acumulación de recursos. Establece un ámbito de discusión fundamental para las ciencias sociales en el país para discutir los procesos de urbanización, sean estos de expansión, de consolidación o de movilidad residencial.

Otra forma de repensar los mecanismos de integración y cohesión social en los barrios populares se encuentra en el trabajo de Franklin Alberto Velarde Herz. Pensar el hábitat popular es poner de relieve la capacidad integradora que tiene la ciudad y las distintas manifestaciones socioespaciales que la componen. En tal sentido, la pregunta fundamental — para este autor — es discutir cómo se produce no solo la vivienda, sino también el espacio público, en tanto lugar de encuentro e interacción. De esta manera, el estudio del espacio público — desde una mirada institucional, etnográfica y urbanística- permite entender cómo se generan los «órdenes sociales» de la vida pública. 
Katherine Sarmiento Viena discute uno de los temas centrales en la configuración de los espacios urbano-populares: las organizaciones sociales de base. Analiza cómo los comedores populares autogestionarios, desde su creación en el año 1979, se convirtieron no solamente en un espacio de subsistencia, sino también en una plataforma de participación de mujeres como líderes a nivel local, al origen de trayectorias diferenciadas que desbordan el propio comedor popular. Así, este artículo forma parte de una tradición relevante en los estudios urbanos que problematizan las relaciones entre vínculos sociales, género y producción social del hábitat. Permite analizar cómo estas configuraciones articulan relaciones asimétricas de poder en su interior.

Los dos últimos artículos tratan de una ciudad secundaria: Huamachuco, en la región norandina del Perú. Alejandro Gonzales Gavilano propone un análisis de la articulación entre dinámicas económicas y urbanización, y de la especialización en función de los espacios urbanos. Discute el rol de la minería en la reestructuración del territorio y del sistema urbano. No solo evidencia cómo se configuran los distintos espacios urbanos de la capital de provincia, sino también cómo los procesos de configuración local responden a un complejo entramado de actividades económicas (como sucede con las actividades extractivas y con las economías delictivas e informales).

Partiendo del mismo estudio de caso, Marta Vilela y Paola Moschella proponen analizar las dinámicas urbanas como parte de un sistema dinámico relacionado con su entorno, no solo los mecanismos de expansión «periférica», sino también las intervenciones sobre el territorio y el paisaje. Las autoras dan cuenta de cómo el Estado, a través de la gestión pública, se convierte en un actor central en la promoción y en la consolidación de un modelo de expansión urbana informal. Analizan los desafíos ambientales, en particular la protección de áreas naturales, frente a una urbanización extensiva. Resaltan la complejidad de la situación de las ciudades intermedias menores donde, por un lado, es necesario facilitar el acceso a la vivienda adecuada y, por otro lado, es importante gestionar y minimizar los impactos ambientales que genera el crecimiento urbano. Estas dinámicas, y en particular la confrontación de acciones y discursos contradictorios, constituyen un tema nuevo en la agenda, en particular en lo que concierne a las ciudades del interior del país.

Las experiencias descritas invitan finalmente a cuestionar la relevancia del caso de Lima como expresión «típica» de los procesos urbanos en el país. Demuestran la necesidad de evitar un traslado de discusiones y explicaciones desde Lima hacia otras partes del Perú urbano, y plantean más bien la necesidad de una agenda de investigación abierta a las diferentes realidades del país. 


\section{Referencias citadas}

BARREDA, J. \& RAMÍREZ CORZO, D., 2004 - Lima: consolidación y expansión de una ciudad popular. In: Perú Hoy: Las ciudades en el Perú (C. E. Aramburú, E. Ballón, J. Barreda, A. Huerta Mercado, M. Llona, D. Ramírez Corzo, G. Riofrío, P. Vega Centeno \& M. Zolezzi): 199-218; Lima: Centro de Estudios y Promoción del Desarrollo (DESCO).

CALDERÓN COCKBURN, J., 2005 - La ciudad ilegal: Lima en el siglo XX, 320 pp.; Lima: Universidad Nacional Mayor de San Marcos.

CALDERÓN, J. \& VEGA CENTENO, P., 2016 - La cuestión urbana en Perú: balance y perspectivas para el siglo XXI. In: La cuestión urbana en la región andina. Miradas sobre la investigación y la formación (P. Metzger, J. Rebotier, J. Robert, P. Urquieta \& P. Vega Centeno, eds.): 175-221; Quito: Pontificia Universidad Católica del Ecuador (PUCE).

DEGREGORI, C. I., BLONDET, C. \& LYNCH, N., 1986 - Conquistadores de un nuevo mundo: de invasores a ciudadanos en San Martín de Porres, 312 pp.; Lima: Instituto de Estudios Peruanos (IEP).

DRIANT, J.-C., 1991 - Las barriadas de Lima, 231 pp.; Lima: Instituto Francés de Estudios Andinos (IFEA), Centro de Estudios y Promoción del Desarrollo (DESCO).

ESPINOZA, A. \& FORT, R., 2017 - Inversión sin planificación: la calidad de la inversión pública en los barrios vulnerables de Lima, 232 pp.; Lima: Grupo de Análisis para el Desarrollo (GRADE).

FERNÁNDEZ-MALDONADO, A. M., 2015 - Las barriadas de Lima como estímulo a la reflexión urbana sobre la vivienda. Revisitando a Turner y de Soto. Wasi: revista de estudios sobre vivienda, vol. 2, n. ${ }^{\circ}$ 3: 7-24. Disponible en https://repository.tudelft. nl/islandora/object/uuid:53578ab9-d734-4d1c-b257-cbc81248cc4c/datastream/ OBJ/download.

MATOS MAR, J., 2004 - Desborde popular y crisis del Estado. Veinte años después, 227 pp.; Lima: Fondo Editorial del Congreso del Perú.

METZGER, P., REBOTIER, J., ROBERT, J., URQUIETA, P. \& VEGA CENTENO, P., eds., 2016 La cuestión urbana en la región andina: Miradas sobre la investigación y la formación, 291 pp.; Quito: Centro de Publicaciones de la Pontificia Universidad Católica del Ecuador (PUCE). Disponible en http://www.ifea.org.pe/libreria/hors-collection/pdf/ cuestion-urbana-region-andina.pdf.

MINISTERIO DE VIVIENDA, CONSTRUCCIÓN Y SANEAMIENTO (MVCS), 2012 Situación de los Barrios Urbano Marginales en el Perú 2012. Segunda aproximación, 121 pp; Lima: Ministerio de Vivienda, Construcción y Saneamiento, Dirección Nacional de Urbanismo.

MOLNÁROVÁ, J., RODRÍGUEZ RIVERO, L., ESPINOZA, A. \& FORT, R., eds., 2017 Otro urbanismo para Lima: más allá del mejoramiento de barrios, 227 pp.; Lima: Fondo Editorial de la Pontificia Universidad Católica del Perú (PUCP), Universidad Científica del Sur, Grupo de Análisis para el Desarrollo (GRADE).

RIOFRÍO, G., 1991 - Producir la ciudad (popular) de los '90: entre el mercado y el Estado, 152 pp.; Lima: Centro de Estudios y Promoción del Desarrollo (DESCO).

SOTO, H. de, 1987 - El otro sendero: La Revolución Informal, 317 pp.; Lima: Instituto Libertad y Democracia.

TURNER, J. \& FICHTER, R., coords., 1976 - Libertad para construir: el proceso habitacional controlado por el usuario, 283 pp.; México, D. F.: Siglo Veintiuno Editores. 